\title{
Stickler Syndrome Type 2
}

National Cancer Institute

\section{Source}

National Cancer Institute. Stickler Syndrome Type 2. NCI Thesaurus. Code C74985.

A rare autosomal dominant syndrome caused by mutations in the COL11A1 gene. It is characterized by an abnormal ocular vitreous architecture (beaded vitreous phenotype). Other signs and symptoms include retinal detachment, joint hypermobility, hearing loss, and midline clefting. 